Article

\title{
Novel Galiellalactone Analogues Can Target STAT3 Phosphorylation and Cause Apoptosis in Triple-Negative Breast Cancer
}

\author{
Hyejin Ko ${ }^{1,+}$, Jong Hyun Lee ${ }^{2,+}{ }^{,}$Hyun Su Kim ${ }^{3}$, Taewoo Kim ${ }^{4}$, Young Taek Han ${ }^{5}$, \\ Young-Ger Suh ${ }^{3}$, Jaemoo Chun ${ }^{1}$, Yeong Shik Kim ${ }^{1, *}$ and Kwang Seok Ahn ${ }^{2, *}$ \\ 1 Natural Products Research Institute, College of Pharmacy, Seoul National University, 1 Gwanak-ro, \\ Gwanak-gu, Seoul 08826, Korea; hyejinko@snu.ac.kr (H.K.); jaemoo.chun@gmail.com (J.C.) \\ 2 Department of Science in Korean Medicine, Kyung Hee University, 24 Kyungheedae-ro, Dongdaemun-gu, \\ Seoul 02447, Korea; jhlee0801@khu.ac.kr \\ 3 College of Pharmacy, CHA University, 120 Haeryong-ro, Pochen-si, Gyenggi-do 11160, Korea; \\ khs8812@snu.ac.kr (H.S.K.); ygsuh@cha.ac.kr (Y.G.S.) \\ 4 College of Pharmacy, Seoul National University, 1 Gwanak-ro, Gwanak-gu, Seoul 08826, Korea; \\ taewookim@snu.ac.kr \\ 5 College of Pharmacy, Dankook University, 119 Dandae-ro, Dongnam-gu, Cheonan 330-714, Korea; \\ hanyt@dankook.ac.kr \\ * Correspondence: ksahn@khu.ac.kr (K.S.A.); kims@snu.ac.kr (Y.S.K.); \\ Tel.: +82-2-961-2316 (K.S.A.); +82-2-880-2479 (Y.S.K.) \\ $\dagger$ These authors contributed equally to this study.
}

Received: 19 March 2019; Accepted: 30 April 2019; Published: 3 May 2019

\begin{abstract}
Aberrant activation of signal transducer and activator of transcription 3 (STAT3) has been documented in various malignancies including triple-negative breast cancers (TNBCs). The STAT3 transcription factor can regulate the different important hallmarks of tumor cells, and thus, targeting it can be a potential strategy for treating TNBC, for which only limited therapeutic options are available. In this study, we analyzed the possible effect of (-)-galiellalactone and its novel analogues, SG-1709 and SG-1721, and determined whether these agents exerted their antineoplastic effects by suppressing the STAT3 signaling pathway in TNBC cells. The two analogues, SG-1709 and SG-1721, inhibited both constitutive as well as inducible STAT3 phosphorylation at tyrosine 705 more effectively than (-)-galiellalactone, which indicates that the analogues are more potent STAT3 blockers. Moreover, SG-1721 not only inhibited nuclear translocation and DNA binding of STAT3 but also induced apoptosis, and decreased expression of diverse oncogenic proteins. Interestingly, SG-1721 also exhibited an enhanced apoptotic effect when combined with radiotherapy. Furthermore, in vivo administration of SG-1721 significantly attenuated breast xenograft tumor growth via decreasing levels of p-STAT3. Therefore, SG-1721 may be a promising candidate for further application as a pharmacological agent that can target STAT3 protein in treating TNBC.
\end{abstract}

Keywords: STAT3; galiellalactone; triple-negative breast cancers (TNBCs); apoptosis; radiation; xenograft

\section{Introduction}

Breast cancer is the most commonly diagnosed cancer among women worldwide [1-6]. There were over two million new cases in 2018 [7-15] and more than 40,000 deaths due to breast cancer each year in the United States [16]. Clinically, breast cancer can be divided into four distinct subtypes, namely: Luminal A, luminal B, human epidermal growth factor receptor 2 (HER2) overexpression, 
and basal-like (Basal A and Basal B) [17]. However, breast cancer is still generally assessed based on the expression of estrogen receptor (ER), progesterone receptor (PR), and HER2 [18]. Triple-negative breast cancer (TNBC) does not express these three molecular markers but exhibits a more aggressive phenotype and has a high risk of recurrence, with mortality ranging from 3 to 5 years after diagnosis with limited treatment options [19]. Therefore, there is a major need to understand the molecular basis of TNBC for the development of effective therapeutic strategies for patients with TNBC. There are many oncogenic molecules that are involved in the progression of breast cancer, and signal transducer and activator of transcription 3 (STAT3) protein can play a multifaceted role in breast tumorigenesis [20-25].

STAT3 protein has been shown to be constitutively active in a large number of tumor tissues, including those derived from breast cancer patients [25-37]. STAT3 can be induced by the leukemia inhibitory factor in normal breast cells, whereas STAT3 is activated by IL-6 stimulation in breast cancer [38]. In addition, intrinsic kinases such as EGFR (epidermal growth factor receptor) and VEGFR (vascular endothelial growth factor receptor) can mediate aberrant phosphorylation of STAT3 in breast cancer [39]. Upon its activation, this transcription factor can regulate proliferation, survival, and metastasis in TNBC and also lead to resistance to chemotherapy as well as radiotherapy [20-25]. Several studies also described that STAT3 was activated in TNBC, suggesting that therapeutic inhibition of STAT3 signaling might be effective in TNBC $[40,41]$. Therefore, finding a potent small molecule which could significantly inhibit STAT3 activation is required.

Galiellalactone (GL), a STAT3 pharmacological inhibitor, is a hexaketide metabolite that can selectively block the binding of active STAT3 dimers to DNA [42]. In spite of tremendous progress in discovery of new synthetic drugs for cancer treatment, application of natural products for targeting aberrant growth and metastasis of tumor cells remains an important therapeutic strategy [43-47]. Moreover, as per the recent reports, GL can significantly attenuate the growth of prostate cancer cells and cause apoptosis [48]. It also inhibits DNA binding of the Smad2/3 transcription factor in both HepG2 as well as MDA-MB-231 cells and also antagonizes the cellular effects of transforming growth factor-beta (TGF- $\beta$ ) [49]. A few prior studies related to the structure-activity relationship of GL have been predominantly performed focused on modifying the basic hydrindane system of GL [50]. Recently, we identified a series of novel STAT3-selective inhibitors based on GL via the truncation of the cyclohexene ring system [51]. Among the structural analogues, SG-1709 and SG-1721 exhibited potent cytotoxicity against TNBC cell lines via the specific inhibitory activity of the phosphorylated STAT3 at the tyrosine 705 residue and selectively inhibited the STAT3 signaling pathway without suppressing the levels of STAT1 and STAT5.

In this study, we examined whether the cyclohexene-truncated bicyclic lactone analogues SG-1709 and SG-1721 derived from natural GL can inhibit tumor growth by effectively blocking the STAT3 signaling pathway in vitro and in vivo in breast cancers. We also investigated whether exposure of GL analogue in conjunction with ionizing radiation can augment apoptosis compared to individual treatment in breast cancer cells.

\section{Materials and Methods}

\subsection{Reagents}

(-)-Galiellalactone and its analogues SG-1709, or SG-1721 were dissolved in DMSO, and the final concentration of DMSO in the cell culture was kept below $0.05 \%$. Dulbecco's modified eagle medium (DMEM), RPMI 1640 medium, fetal bovine serum (FBS), antibiotic-antimycotic mixture, and LightShift ${ }^{\circledR}$ Chemiluminescent EMSA kit were obtained from Thermo Fisher Scientific Inc. (Waltham, MA, USA). 5'-biotinylated STAT3 was obtained from Bioneer Corporation (Daejeon, Korea). 3-(4,5-Dimethylthiazol-2-yl)-2,5-diphenyltetrazolium bromide (MTT) was purchased from Sigma Aldrich (St. Louis, MO, USA). Epidermal growth factor (EGF) and IL-6 were purchased from R\&D Systems (Minneapolis, MN, USA). Antibodies against STAT3, Lamin B, Bcl-xL, Bcl-2, Cyclin D1, MMP-9, PARP (Poly (ADP-ribose) polymerase), Caspase-3, MMP-2, COX-2, Ki-67, and $\beta$-actin were purchased 
from Santa Cruz Biotechnology (Santa Cruz, CA, USA); p-STAT3(Tyr705), p-JAK1(Tyr1022/1023), JAK1, p-JAK2 (Tyr1007/1008), JAK2, Cleaved caspase-3, and Cyclin D1 were purchased from Cell signaling Technology (Beverly, MA, USA).

\subsection{Cell Lines}

Human triple-negative breast cancer cell lines (BT-549, BT-20, and MDA-MB-468), luminal A breast cancer cell lines (MCF-7 and T47D), HER2 breast cancer cell lines (SK-BR-3 and MDA-MB-453), and nontumorigenic epithelial cell lines MCF-10A were purchased from the American Type Culture Collection (Manassas, VA, USA). The MDA-MB-468 and MDA-MB-453 cells were grown in DMEM supplemented with $10 \%$ FBS and $1 \%$ antibiotics (penicillin $100 \mathrm{U} / \mathrm{mL}$ and streptomycin $100 \mu \mathrm{g} / \mathrm{mL}$ ).

\subsection{Western Blot Assay}

MDA-MB-468 cells were seeded in 6-well plates at a density of $1 \times 10^{6}$ cells/well. The cells were treated with the indicated concentrations ( 5 or $10 \mu \mathrm{M}$ ) of (-)-galiellalactone (GL), its active analogues SG-1709, or SG-1721 for 4 or $24 \mathrm{~h}$. Whole cell lysates were prepared using a lysis buffer (20 mM HEPES, pH 7.6, $350 \mathrm{mM} \mathrm{NaCl}$, 20\% glycerol, $0.5 \mathrm{mM}$ EDTA, $0.1 \mathrm{mM}$ EGTA, 1\% NP-40, $50 \mathrm{mM} \mathrm{NaF}, 1 \mathrm{mM}$ DTT, 1 mM PMSF, protease inhibitor cocktail, and phosphatase inhibitor cocktail). The lysates were centrifuged, and the supernatant was collected. Equal amounts of protein were resolved on 8-15\% SDS-PAGE and transferred to a nitrocellulose membrane. The membrane was blocked with $5 \%$ bovine serum albumin (BSA) and incubated with primary antibodies overnight at $4{ }^{\circ} \mathrm{C}$. The blots were washed and incubated with secondary antibodies conjugated with HRP (horseradish peroxidase) for $1 \mathrm{~h}$ and then examined by chemiluminescence (Intron Biotechnology, Seoul, Korea) and visualized using a LAS-1000 image analyzer (Fujifilm, Tokyo, Japan).

\subsection{Immunocytochemistry for p-STAT3 and STAT3 Localization}

The MDA-MB-468 cells were plated on coverslips and allowed to attach by overnight incubation. The cells were treated with $10 \mu \mathrm{M}$ of GL, SG-1709, or SG-1721 for $4 \mathrm{~h}$ and fixed with $4 \%$ paraformaldehyde (PFA) for $20 \mathrm{~min}$ at room temperature. The cells were permeabilized with $0.2 \%$ Triton X-100 in PBS (phosphate buffered saline) for 20 min. After a brief washing in PBS, slides were blocked with $5 \%$ bovine serum albumin (BSA) for $1 \mathrm{~h}$ at room temperature, then incubated either with goat polyclonal anti human p-STAT3 antibody (dilution, 1:100) or with rabbit polyclonal anti human STAT3 antibody (dilution, 1:100) overnight at $4{ }^{\circ} \mathrm{C}$. The slides were washed and then incubated either with Alexa Fluor 488 (dilution, 1:1000) antigoat IgG1 or with Alexa Fluor 594 (dilution, 1:1000) antirabbit IgG1 for $1 \mathrm{~h}$ at room temperature in the dark. Next, counterstained for nuclei with $5 \mu \mathrm{g} / \mathrm{mL}$ DAPI (4',6-diamidino-2-phenylindole) solution for $3 \mathrm{~min}$. Stained slides were mounted with mounting medium (GBI Laboratories, Manchester, UK) and analyzed under an Olympus FluoView FV1000 confocal microscope (Tokyo, Japan). DAPI and FITC (fluorescein-5-isothiocyanate) fluorescence were excited (Ex: $405 \mathrm{~nm}$ and $488 \mathrm{~nm}$ ) and detected (Em: $461 \mathrm{~nm}$ and $519 \mathrm{~nm}$ ) with $2.1 \%$ laser transmissivity and $5.0 \%$ laser transmissivity, respectively.

\subsection{Electrophoretic Mobility Shift Assay (EMSA) for STAT3-DNA Binding}

STAT3-DNA binding was analyzed by an electrophoretic mobility shift assay (EMSA) using a 5'-biotinylated STAT3 oligonucleotide (5'-GATCCTTCTGGGAATTCCTAGATC-3' and 5'-GATCTAGGAATTCCCAGAAGGATC-3'). Briefly, nuclear extracts were prepared from GL, SG-1709, or SG-1721-treated cells and incubated with the 5'-biotinylated STAT3 oligonucleotide probes. The DNA-protein complex formed was separated from free oligonucleotide on $6 \%$ native polyacrylamide gels and transferred to a positively charged nylon membrane. The membrane was

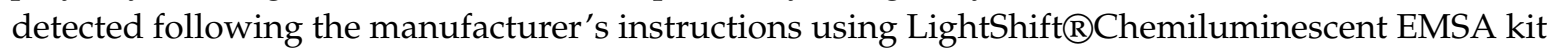
(Waltham, MA, USA). 


\subsection{STAT3-Dependent Luciferase Reporter Assay}

MDA-MB-468 cells were plated in 24 -well plates at a density of $1 \times 10^{5}$ cells/well. After $24 \mathrm{~h}$, the cells were transiently transfected with a p-STAT3-Luc reporter vector in the presence of a pCMV-Luc vector (Firefly) using a transfection reagent (Intron Biotechnology, Seoul, Korea). At $24 \mathrm{~h}$ post-transfection, the cells were pretreated with $5 \mu \mathrm{M}$ of GL, SG-1709, or SG-1721 for $24 \mathrm{~h}$ and then induced by IL-6 or EGF for additional 5 or $30 \mathrm{~min}$. The luciferase assay was performed using the Dual Luciferase Reporter Assay System (Promega, Madison, WI, USA) in accordance with the manufacturer's instructions. The luminescence signal was measured using a luminometer (MicroLumat Plus, Berthold Technologies, Dortmund, Germany).

\section{7. si-RNA Trnasfection}

To test whether our drugs are really functioning through STAT3, transient transfections were carried out using STAT3 (Santa Cruz, CA, USA) and scrambled si-RNAs (Bioneer Corporation, Daejeon, Korea). MDA-MB-468 cells were plated in 24 -well plates at a density $1 \times 10^{5}$ cells/well. After $24 \mathrm{~h}$, the cells were transiently transfected with STAT3 and scrambled (control) si-RNAs using a transfection reagent (Intron Biotechnology, Seoul, Korea). At $24 \mathrm{~h}$ post-transfection, the cells were treated with $10 \mu \mathrm{M}$ of SG-1721 for $4 \mathrm{~h}$ or $24 \mathrm{~h}$ and then performed Western blot assay or annexin $\mathrm{V}$ assays.

\subsection{Cell Cycle Analysis}

To determine apoptosis, cell cycle analysis was performed using propidium iodide. MDA-MB-468 cells were plated in 6-well plates at a density of $1 \times 10^{6}$ cells/well. After treatment with $10 \mu \mathrm{M}$ of SG-1721 for $24 \mathrm{~h}$, the cells were collected and washed with $1 \times$ PBS. Cell pellets were fixed in $70 \%$ cold ethanol overnight at $-20^{\circ} \mathrm{C}$. The fixed cells were resuspended in $1 \times$ PBS containing $1 \mathrm{mg} / \mathrm{mL}$ RNase A, incubated for $1 \mathrm{~h}$ at $37^{\circ} \mathrm{C}$ incubation. Cells were then washed, resuspended, and stained in PBS containing $25 \mu \mathrm{g} / \mathrm{mL}$ of propidium iodide for $30 \mathrm{~min}$ at room temperature in the dark. The DNA contents of the stained cells were analyzed using Cell Quest Software with a FACScan Calibur flowcytometry (BD Biosciences, Becton-Dickinson, Franklin Lakes, NJ, USA).

\subsection{Annexin $V$ Assays}

MDA-MB-468 cells were seeded in plat and treated with $10 \mu \mathrm{M}$ of SG-1721 for $24 \mathrm{~h}$. Apoptosis was evaluated by annexin V-FITC and Propidium Iodide stained cells using FITC Annexin V Apoptosis Detection Kit I in accordance with the manufacturer's protocols. Briefly, the cells were harvested using $1 \%$ trypsin in PBS (welgene, Gyeongsangbuk-do, Korea), washed once with cold PBS. The cell pellet was resuspended in $1 \times$ binding buffer add $5 \mu \mathrm{L}$ of FITC Annexin V and $5 \mu \mathrm{L}$ of propidium iodide (PI) for $15 \mathrm{~min}$ at room temperature in the dark. Stained samples were analyzed by FACScan Calibur flowcytometry (BD Biosciences, Becton-Dickinson, Franklin Lakes, NJ, USA).

\subsection{TUNEL Assays}

Individual apoptotic cell death was observed using a Roche Diagnosis TUNEL (Terminal transferase mediated dUTP-fluorescein nick end labeling) assay kit in accordance with the manufacturer's instructions. Briefly, cells treated with $10 \mu \mathrm{M}$ of SG-1721 for $24 \mathrm{~h}$ were washed with cold PBS. The cells were fixed with $4 \%$ paraformaldehyde for $30 \mathrm{~min}$ and washed twice with PBS. The resuspended cells were put in a permeabilization solution $(0.1 \%$ Triton X-100 and $0.1 \%$ Sodium citrate) for $20 \mathrm{~min}$ at $4{ }^{\circ} \mathrm{C}$, and then the cells were washed with cold PBS. The cells were subsequently incubated with a TUNEL enzyme and TUNEL label mixture for $1 \mathrm{~h}$ at $37^{\circ} \mathrm{C}$ in a humidified atmosphere in the dark. After being washed with PBS, cells were analyzed using FACScan Calibur flowcytometry (BD Biosciences, Becton-Dickinson, Franklin Lakes, NJ, USA). 


\subsection{Cytotoxicity and Combination Index}

To measure cytotoxicity, MTT assay was carried out as previously described [52]. To analyze the combination index, the effects of drug combinations were evaluated with Calcusyn software (Biosoft, Cambridge, UK), which employs the Chou-Talalay combination index method, which is based on the median-effect equation, itself a derivation from the mass-action law [53].

\subsection{Irradiation and Cytotoxicity Assay}

To measure cytotoxicity, MTT assay was carried out as previously described [52]. To analyze the combination index, the effects of drug combinations were evaluated with Calcusyn software (Biosoft, Cambridge, UK). This software uses the Chou-Talalay combination index method, which is based on the median-effect equation, itself a derivation from the mass-action law [53]. We entered the resulting data, along with the data obtained from single drug treatments, into Calcusyn to determine a combination index value $(\mathrm{CI})$ for each combination point, which quantitatively defines additivity $(\mathrm{CI}=1)$, synergy $(\mathrm{CI}<1)$, and antagonism $(\mathrm{CI}>1)$.

\subsection{Animals}

All procedures involving animals were reviewed and approved by Seoul National University Institutional Animal Care and Use committee (SNU-160908-1). Six-week-old athymic nu/nu female mice were purchased form Nara Biotec CO (Gyeonggi-do, Korea).

\subsection{In Vivo Studies}

When tumors had reached $0.25 \mathrm{~cm}$ in diameter, the mice were randomized into the following treatment groups ( $n=6$ /group) based on the tumor volume. Group I (control) was treated with PBS and group II was treated with SG-1721 $(0.5 \mathrm{mg} / \mathrm{kg})$. Treatment was continued for up to 20 days from the date of randomization (Day 0 ). The tumors were carefully excised and measured as carried out previously [37].

\subsection{Immunohistochemical Analysis of Tumor Samples}

Solid tumors from control and treatment groups were fixed with $10 \%$ neutral buffered formalin (BBC Biochemical, USA), processed and embedded in paraffin. Sections were cut and deparaffinized in xylene and dehydrated in graded alcohol and finally hydrated in water. Antigen retrieval was performed by boiling the slide in $10 \mathrm{mM}$ sodium citrate ( $\mathrm{pH}$ 6.0) for $30 \mathrm{~min}$. Immunohistochemistry was performed following manufacturer instructions (Vector Laboratories ImmPRESSTM REAGENT KIT). Briefly, endogenous peroxidases were quenched with $3 \%$ hydrogen peroxide. Nonspecific binding was blocked by incubation in the blocking reagent in the ImmPRESSTM REAGENT KIT (Vector Laboratories, Burlingame, CA, USA) in accordance with the manufacturer's instructions. Sections were incubated overnight with primary antibody: p-STAT3, anti-Ki-67, and anticleaved caspase-3 (at 1:100 dilutions). Slides were subsequently washed several times in PBS and were incubated with ImmPRESSTM reagent in accordance with the manufacturer's instructions. Immunoreactive species were detected using 3, 3-diaminobenzidine tetrahydrochloride (DAB) as a substrate. Sections were counterstained with Gill's hematoxylin and mounted under glass cover slips. Images were taken using a Nikon Eclipse Ts2 inverted routine microscope (magnification, 40×).

\subsection{Statistical Analysis}

All the data are presented as the mean \pm standard deviation (SD) from at least three independent experiments. An analysis of variance (ANOVA) with the Dunnett's t-test was used for the statistical analysis of multiple comparisons. A value of $p<0.05$ was chosen as the criterion for statistical significance. 


\section{Results}

\subsection{GL and Its Analogues Block Constitutive Phosphorylation of STAT3 in TNBC Cells}

The chemical structures of GL and its analogues, SG-1709 and SG-1721, are shown in Figure 1A. Firstly, we examined the phosphorylation status of STAT3 in different subtypes of breast cancer cells. We observed that STAT3 was persistently phosphorylated in TNBC cells BT-549, BT-20, and MDA-MB-468 cells. By contrast, STAT3 was expressed at very low levels in luminal A (MCF-7 and T-47D) and HER2 (SK-BR-3 and MDA-MB-453) breast cancer cells (Figure 1B). We next elucidated whether GL and its novel analogues can modulate STAT3 activation, on TNBC cells. It was found that the two analogues, SG-1709 and SG-1721, inhibited both constitutive and inducible STAT3 phosphorylation at tyrosine 705 more effectively than GL (Figure 1C). Moreover, as shown in Figure 1D, STAT3 phosphorylation was suppressed in MDA-MB-468 cells in a time-dependent manner, and SG-1721 most effectively inhibited STAT3 phosphorylation.

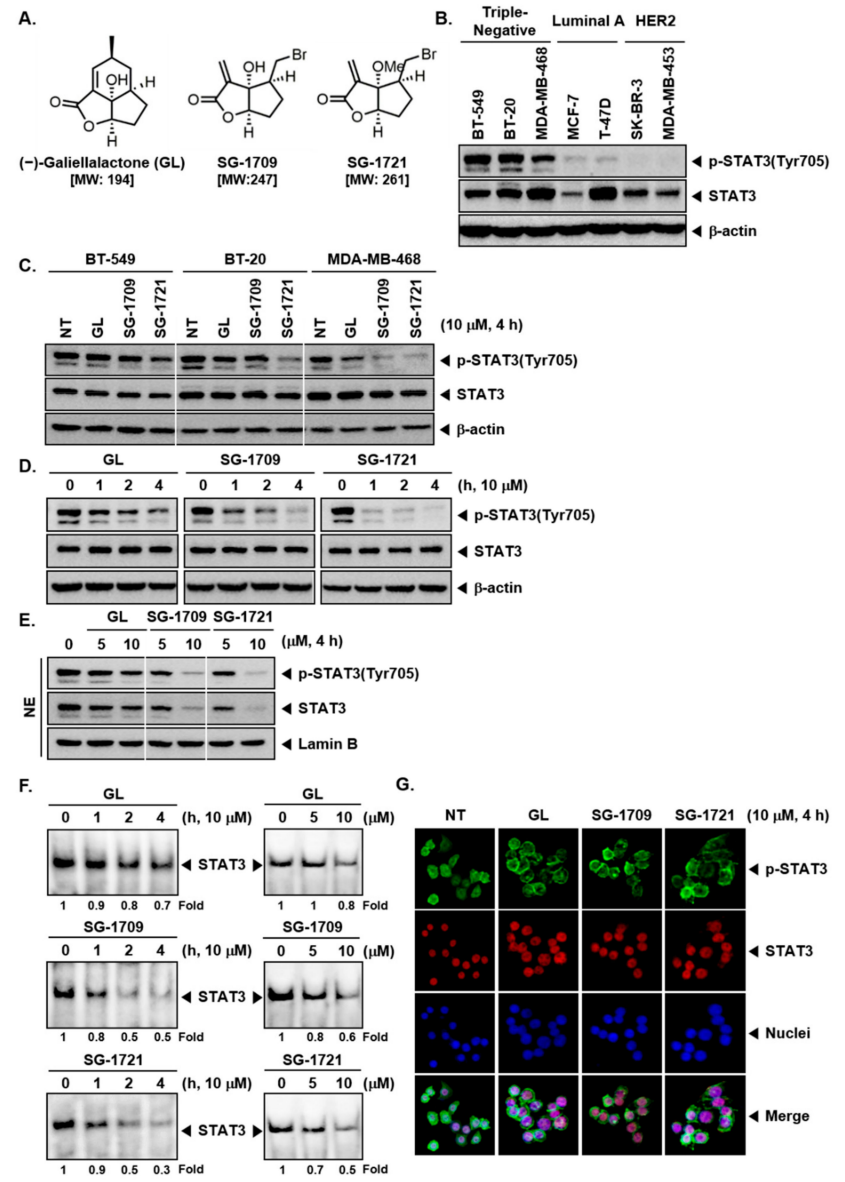

Figure 1. (-)-Galiellalactone (GL) and its analogues can block the STAT3 signaling pathway. (A) The chemical structure of (-)-galiellalactone (GL), SG-1709, and SG-1721. (B) Detection levels of p-STAT3 and STAT3 in seven human breast cancer cell lines were analyzed by Western blot analysis. (C) BT-549, BT-20, and MDA-MB-468 cells were treated with $10 \mu \mathrm{M}$ of GL, SG-1709, and SG-1721 for $4 \mathrm{~h}$, and Western blot analysis was done for different proteins. (D) MDA-MB-468 cells as described above for various time intervals and Western blot analysis was done. (E) MDA-MB-468 cells were treated with concentrations of GL, SG-1709, and SG-1721 for $4 \mathrm{~h}$, and Western blot analysis was done. (F) MDA-MB-468 cells were treated as described above in panel D and E, and electrophoretic mobility shift assay was done. (G) MDA-MB-468 cells were treated with $10 \mu \mathrm{M}$ of GL, SG-1709, and SG-1721 for $4 \mathrm{~h}$, and immunocytochemistry was done. 


\subsection{GL and Its Analogues Act at Multiple Steps in STAT3 Signaling Pathway}

It was deciphered next whether GL, SG-1709, and SG-1721 could also affect the nuclear translocation of STAT3. It was noted that $5 \mu \mathrm{M}$ of GL, SG-1709, and SG-1721 slightly suppressed the translocation of STAT3 to the nuclei, but $10 \mu \mathrm{M}$ of SG-1709 and SG-1721 inhibited this translocation of STAT3 into the nuclei more potently as compared to GL (Figure 1E). Next, it was confirmed by electrophoretic mobility shift assay (EMSA) that GL, SG-1709, and SG-1721 decreased STAT3-DNA binding activity in a time- and concentration-dependent manner, among which SG-1721 was most effective (Figure 1F). Furthermore, using immunocytochemistry, it was demonstrated that GL, SG-1709, and SG-1721 reduced the translocation of p-STAT3 and STAT3 to the nucleus in MDA-MB-468 cells (Figure 1G).

\subsection{GL And Its Analogues Inhibit Inducible STAT3 Phosphorylation}

We also examined whether GL, SG-1709, and SG-1721 can also abrogate IL-6 or EGF-induced STAT3 phosphorylation in MDA-MB-468 cells. Results show that IL-6 $(25 \mathrm{ng} / \mathrm{mL})$ or EGF $(50 \mathrm{ng} / \mathrm{mL})$ caused substantial STAT3 activation and GL, SG-1709, and SG-1721 exposure inhibited inducible STAT3 phosphorylation. (Figure 2A,C). We also noted that upon pretreatment with GL, SG-1709, and SG-1721, IL-6 or EGF-induced STAT3 luciferase activity was inhibited and the two analogues were more effective than GL (Figure 2B,D).

A.

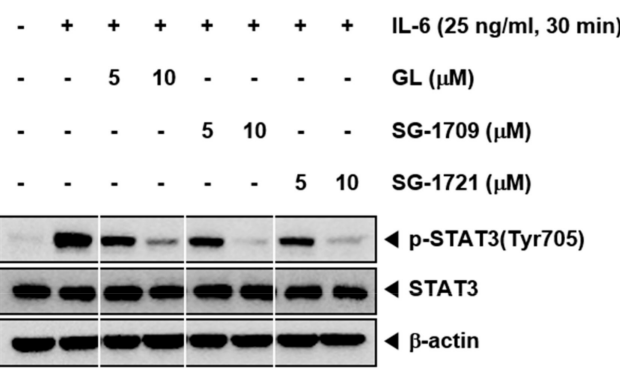

c.

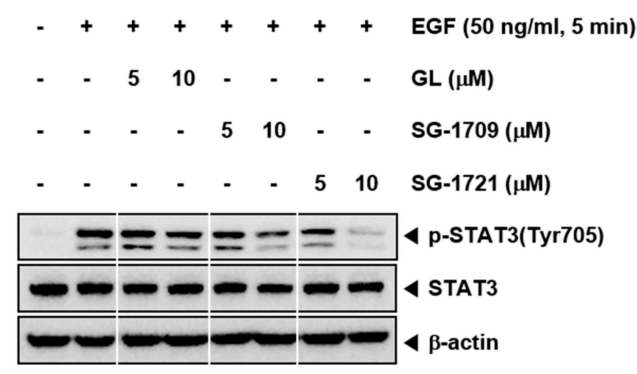

E.

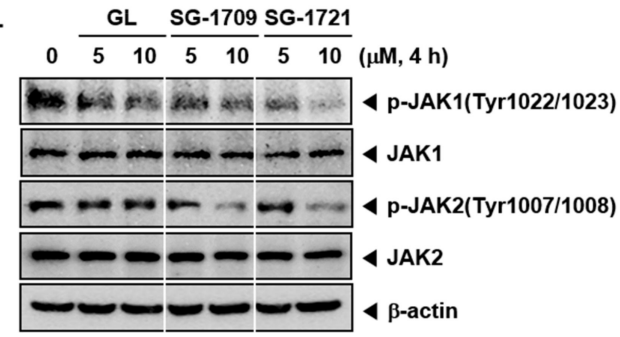

B.

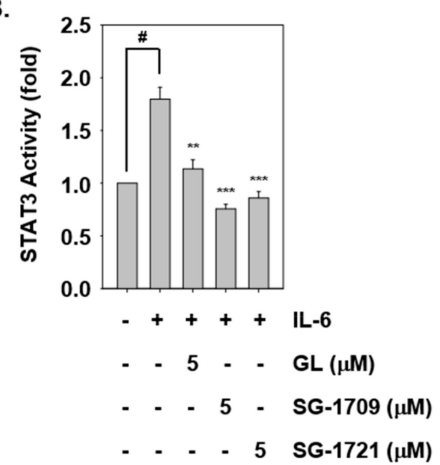

D.

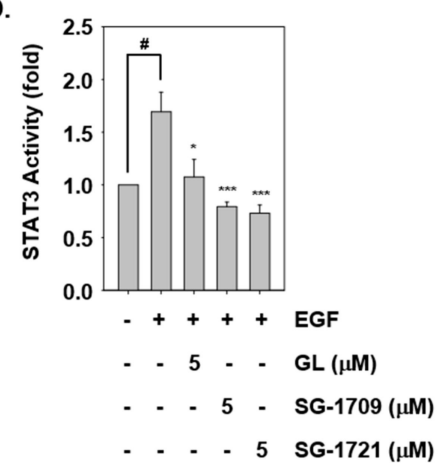

Figure 2. GL and its analogues inhibit inducible STAT3 activation. (A) MDA-MB-468 cells were pretreated with concentrations of GL, SG-1709, and SG-1721 for $4 \mathrm{~h}$, then simulated with IL- 6 . Thereafter, equal amounts of lysates were analyzed by Western blot analysis using antibodies against p-STAT3 
(Tyr705) and STAT3. (B) MDA-MB-468 cells were treated as described above in panel A, and luciferase activity was measured. The results shown are representative of three independent experiments. $\# p<0.001{ }^{*} p<0.05,{ }^{* *} p<0.01 ;{ }^{* * *} p<0.001$. (C) MDA-MB-468 cells were pretreated as described above and then simulated with epidermal growth factor (EGF). Thereafter, Western blot analysis was carried out. (D) MDA-MB-468 cells were treated as described above in panel C, and luciferase activity was measured. The results shown are representative of three independent experiments. \# $p<0.001$; ${ }^{*} p<0.05,{ }^{* * *} p<0.001$. (E) MDA-MB-468 cells were treated with concentrations of GL, SG-1709, and SG-1721 for $4 \mathrm{~h}$, and Western blot analysis was done.

\subsection{GL and Its Analogues Reduce JAK1/2 Phosphorylation in MDA-MB-468 Cells}

We next analyzed the effects of GL, SG-1709, and SG-1721 on JAK1 and JAK2 activation. As shown in Figure 2E, JAK1 and JAK2 were constitutively phosphorylated in MDA-MB-468 cells. Although treatment with $5 \mu \mathrm{M}$ of GL, SG-1709, and SG-1721 did not completely block constitutive JAK1/2 activation $10 \mu \mathrm{M}$ of SG-1709 and SG-1721 inhibited these activation more potently than GL. However, GL could only weakly suppress JAK1 and JAK2 phosphorylation.

\subsection{GL and Its Analogues Suppress Cell Viability and Proliferation}

Next, we proceeded to test whether GL and its analogues can also exert cytotoxic effects against TNBC MDA-MB-468 cells and breast epithelial MCF-10A cells. Interestingly, it was noted that the cell viability of tumor cells was reduced to the maximum level at $10 \mu \mathrm{M}$ dose of SG-1721 among the others analyzed. By contrast, all had a relatively minor effect on the viability of normal cell line MCF-10A (Figure 3A). In addition, it was also observed that GL and its analogues significantly inhibited cell proliferation, and SG-1721 treatment inhibited cell proliferation at the highest rate (Figure 3B).

\subsection{SG-1721 Can Induce Cell Cycle Arrest and Promote Apoptosis}

We performed multiple biochemical assays to determine the effect of the SG-1721 on apoptosis. As depicted in Figure 3C, it was observed that cells accumulated to a greater extent in the $S$ phase upon drug exposure. We next analyzed the effect of SG-1721 on annexin V binding and noted that the treatment caused the percentage of apoptotic cells to be higher than in nontreated cells, increasing from $2.5 \%$ to $12.6 \%$ (Figure 3D). The percentage of cells that underwent apoptosis also increased significantly after treatment with SG-1721, from $2.7 \%$ to $17.4 \%$, as observed following TUNEL assay (Figure 3E). Further, as shown in Figure 3F, a substantial activation of caspase-3 and PARP cleavage was noted upon SG-1721 exposure. Additionally, Western blot analysis showed that SG-1721 can also markedly reduce the expression of Bcl-xL, Bcl-2, Cyclin D1, MMP-9, MMP-2, and COX-2 proteins (Figure 3G). We next tested whether SG-1721 induces cell apoptosis through inhibiting the STAT3 pathway, and transient transfections were carried out using STAT3 and scrambled si-RNA (control) in MDA-MB-468 cells. As shown in Figure 3H, phosphorylated and total STAT3 expression were substantially blocked upon transfection with STAT3/si-RNA. Subsequently, we observed that the knockdown of STAT3 with STAT3/si-RNA reversed the proapoptotic effects induced by SG-1721. The fold change in apoptotic cells was reduced from $8.5 \%: 1.5 \%$ to $3.9 \%: 1.7 \%$ (Figure $3 \mathrm{I}$ ). 
A.
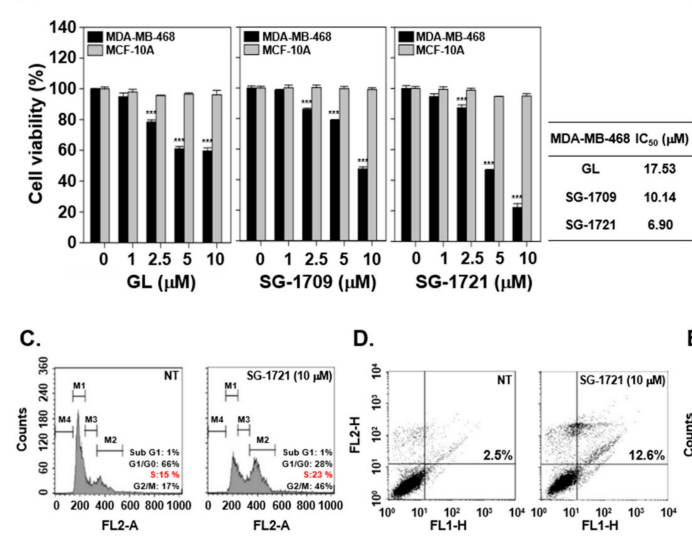

D.

D.
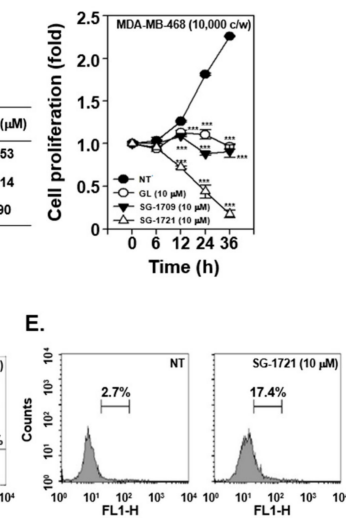

F.

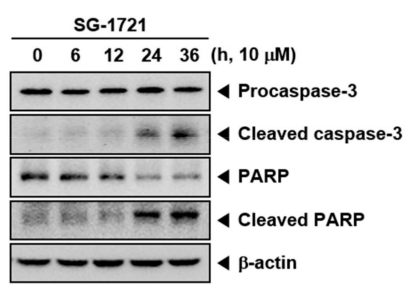

G.

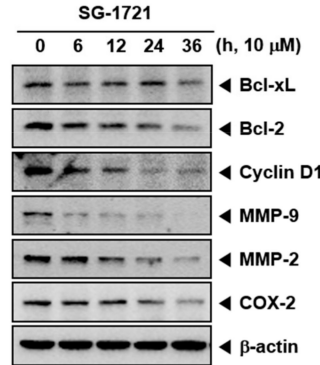

H.
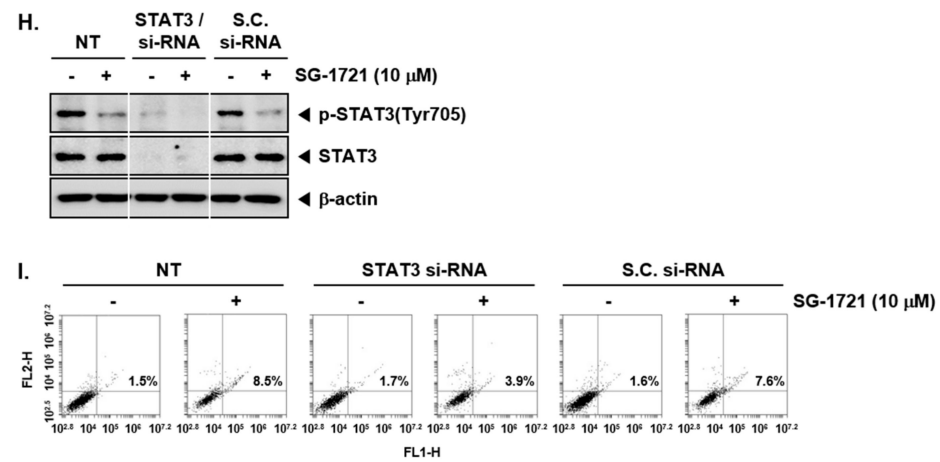

Figure 3. GL and its analogues can cause apoptosis. (A) MDA-MB-468 and MCF-10A cells were treated with various concentrations of GL, SG-1709, and SG-1721 for $24 \mathrm{~h}$, and cell viability was determined using the MTT (3-(4,5-Dimethylthiazol-2-yl)-2,5-diphenyltetrazolium bromide) assay (left panel). $\mathrm{IC}_{50}$ values were determined as the drug concentration at $50 \%$ inhibition of the cell viability (right panel). ${ }^{* *} p<0.001$. (B) MDA-MB-468 cells $(10,000$ cells/wells) were seeded for overnight and then treated with $10 \mu \mathrm{M}$ of GL, SG-1709, and SG-1721, and cell proliferation assay was done. *** $p<0.001$. (C) MDA-MB-468 cells were treated with $10 \mu \mathrm{M}$ of SG-1721 for $24 \mathrm{~h}$. Cellular DNA staining incorporating propidium iodide (PI) and flow cytometry analysis was carried out to determine the cell-cycle distribution. (D) MDA-MB-468 cells were treated as described in panel C, stained with FITC (fluorescein-5-isothiocyanate)-conjugated anti-Annexin V, and flow cytometer was used for analysis. (E) MDA-MB-468 cells were treated as described in panel C, and terminal transferase mediated dUTP-fluorescein nick end labeling (TUNEL) staining was performed (F-G) MDA-MB-468 cells were treated with $10 \mu \mathrm{M}$ of GL, SG-1709, and SG-1721 for various intervals, and Western blot analysis was done. (H) MDA-MB-468 cells were transiently transfected with STAT3 or scrambled si-RNA (control). Then, the transfected cells were treated with $10 \mu \mathrm{M}$ of SG-1721 for $4 \mathrm{~h}$. Thereafter, Western blot analysis was carried out. (I) The transfected MDA-MB-468 cells were treated with $10 \mu \mathrm{M}$ of SG-1721 for $24 \mathrm{~h}$, and then annexin V assays was carried out as described above in panel D. 


\subsection{SG-1721 Can Sensitize TNBC Cells to Radiotherapy}

The enhancement effect of SG-1721 on the radio-sensitivity of MDA-MB-468 cells was analyzed by MTT assay following treatment with various concentrations of SG-1721 $(0.5,1$, or $1.5 \mu \mathrm{M})$ after irradiation with different doses of IR $(1,5$, or $10 \mathrm{~Gy})$. According to the results, the highest synergic cytotoxicity was obtained when the combined treatment of $0.5 \mu \mathrm{M}$ of SG-1721 and 10 Gy of IR was used. (Figure 4A, left panel). The combination index (CI) indicated that effects are prominent at a combination dose of $0.5 \mu \mathrm{M}$ of SG-1721/10 Gy of IR, which synergistically inhibited growth (Figure 4A, right panel). Moreover, as shown in Figure 4B, SG-1721 and IR (10 Gy) treatment alone showed minimal effects on the levels of p-STAT3 as well as p-JAK-1/2, while the combination treatment clearly suppressed activation of these oncogenic proteins in MDA-MB-468 cells. Further, as shown in Figure 4C, SG-1721 or IR (10 Gy) alone showed minimal effects the expression of Bcl-2, Cyclin D1, MMP-2, and COX-2 proteins. However, the combination treatment substantially reduced the expression of these proteins. We next investigated whether caspase activities are required to enhance SG-1721-mediated radiosensitive apoptosis. Combination treatment of SG-1721 and IR showed that cleavage of PARP and the expression of cleaved caspase-3 was found to be increased (Figure 4D). These results provide distinct evidence that SG-1721 can indeed sensitize TNBC cells exposed to radiation, and inhibition of p-STAT3 may play a key role in inducing this effect.

A.
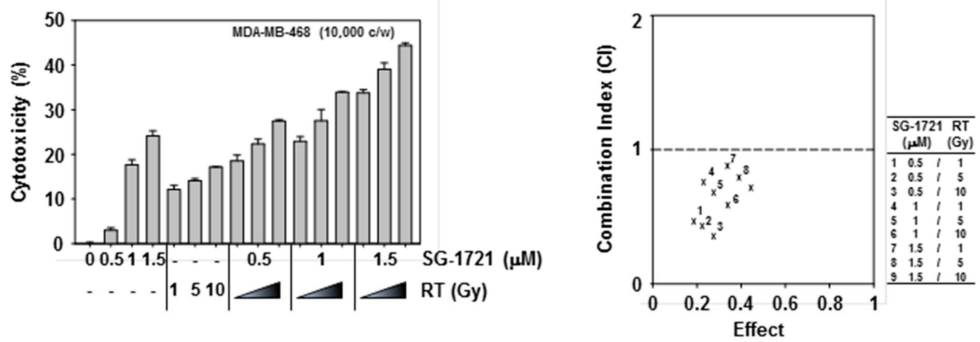

B.

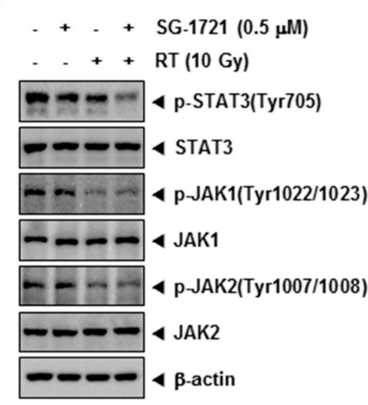

D.

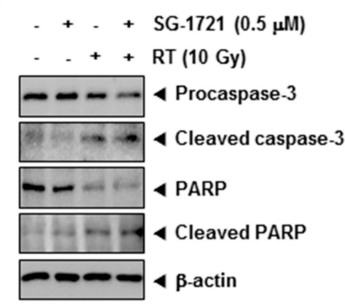

c.

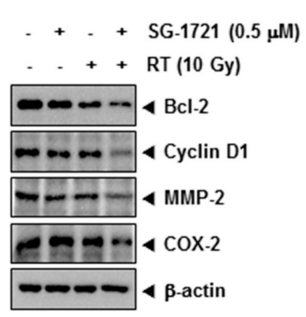

Figure 4. The effect of SG-1721 in combination with radiation on STAT3 signaling cascade (A) MDA-MB-468 cells treated with irradiation (1, 5, or $10 \mathrm{~Gy}$ ) after $4 \mathrm{~h}$ of pretreatment with SG-1721 $(0.5,1$, or $1.5 \mu \mathrm{M})$ incubated for $20 \mathrm{~h}$. The cytotoxicity was determined by MTT assays (left panel). SG-1721 synergistically enhances radiation-induced cell death in MDA-MB-468 cells (right panel). The average of the CI values was obtained at nine different combinations. (B) MDA-MB-468 
cells were treated with $0.5 \mu \mathrm{M}$ of SG-1721 or 10 Gy of irradiation alone or in combination for $4 \mathrm{~h}$. Thereafter, Western blot analysis was performed. (C) MDA-MB-468 cells were treated with irradiation (10 Gy) after $4 \mathrm{~h}$ of pretreatment with SG-1721 $(0.5 \mu \mathrm{M})$ and incubated for $20 \mathrm{~h}$ followed by Western blot analysis. (D) MDA-MB-468 cells were treated as described above in panel C, and Western blot was performed using various antibodies.

\subsection{SG-1721 Can Inhibit Tumor Growth and Alter the Expression of Oncogenic Biomarkers In Vivo}

The preclinical effects of SG-1721 were analyzed as per the protocol depicted in Figure 5A. We noted that the treatment with SG-1721 resulted in a significant suppression of tumor size following drug exposure (Figure 5B). The tumor growth in the SG-1721 group was significantly more abrogated than in the vehicle group (Figure 5C,D) with an increase in the body weight of the mice (Figure 5E). We also noticed that SG-1721 was effective in reducing the expression of various proteins, including p-STAT3 in tumor tissues (Figure 5F). Interestingly, we further observed that SG-1721 substantially suppressed STAT3 phosphorylation, proliferation marker Ki-67, and increased cleaved caspase-3 levels in tumor tissues (Figure 5G), thus depicting the potent anticancer effects of SG-1721 in vivo.

A.

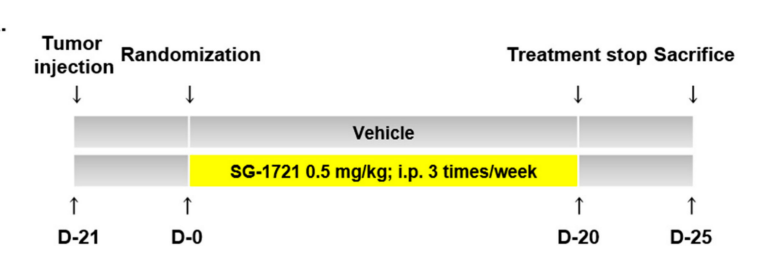

B.

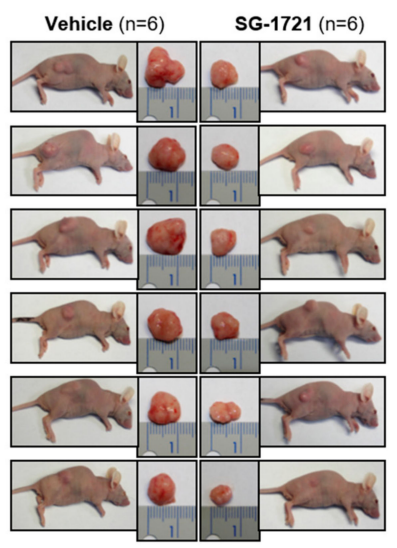

E.

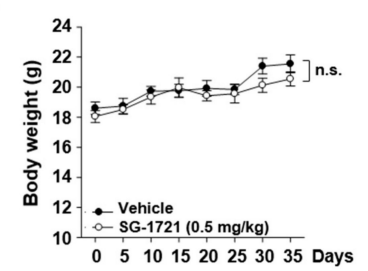

G.

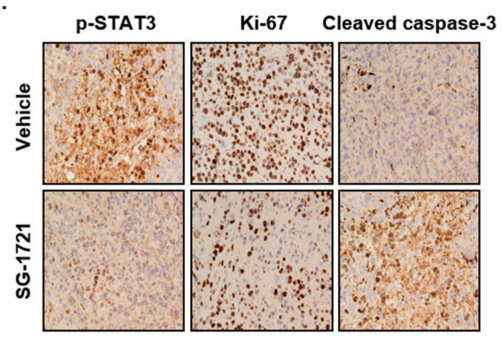

C.

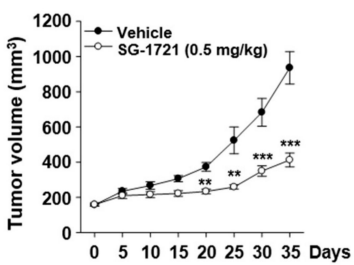

D.

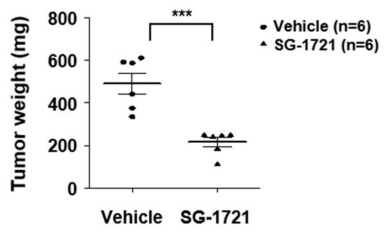

F.
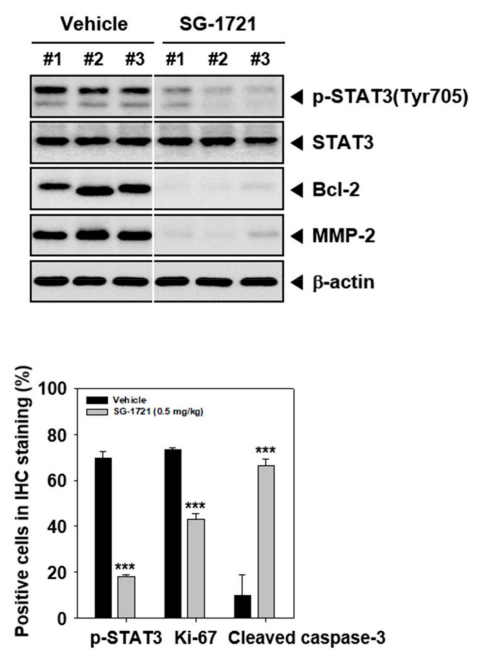

Figure 5. SG-1721 inhibits tumor growth in vivo. (A) A schematic representation of experimental protocol described in "Materials and Methods". (B) Necropsy photographs of mice bearing 
subcutaneously implanted triple-negative breast cancer (TNBC) cells. (C) The tumor diameters were measured at 5-day intervals, and the tumor volumes were calculated using the formula $V=4 / 3 \pi \mathrm{r}^{3}$ $(n=6)$. (D) Tumor weight was measured during the experiment. (E) Body weight changes of mice were measured at indicated times. (F) Western blot of various proteins of interest was carried out in lysate from vehicle control and SG-1721 treated mice. (G) Immunohistochemical analysis of p-STAT3, proliferation marker Ki-67, and cleaved caspase-3 in the tumor tissues (left panel). The results shown are representative of the three independent experiments. Graphs represent positive cells in IHC staining (right panel).

\section{Discussion}

The focus of this study was to analyze the activity of GL analogues against tumor growth in TNBC cell lines and a preclinical model. We observed that GL analogue can be more effective than GL in exerting their anticancer effects through targeted abrogation of the STAT3 mediated signal transduction cascade.

We first measured STAT3 expression levels in several molecular subtypes of breast cancer cell lines and observed that STAT3 was phosphorylated persistently in TNBC cell lines. TNBC is an aggressive disease with poor prognosis because there are no specific treatment options for it. Thus, inhibition of phosphorylated STAT3 may be a potential strategy for the treatment of TNBC. We evaluated the effects of GL and GL analogues SG-1709 and SG-1721 on the STAT3 phosphorylation at Tyr705 residues using diverse assays. We also determined in detail the mechanisms by which GL and GL can negatively regulate STAT3 activation in TNBC cells.

STAT3 is active constitutively in a variety of human cancer cells and tissues, including multiple myeloma and lung cancer [29,54]. It is considered to be an oncogene because it has the ability to promote malignant tumors [55]. STAT3 regulates the expression of various genes in response to cell stimulation and plays an important role in many cell processes, such as cell growth and apoptosis [56]. STAT3 is phosphorylated by JAK in response to ligands such as interferon, epidermal growth factor (EGF), IL-6, and translocation to cell nucleus acting as transcription activators [57]. In addition to direct activation of STAT3 protein by receptor tyrosine kinases, it can also be activated via upstream kinases such as JAKs [58]. The activity of JAK is induced when a regulatory molecule binds and two receptor molecules form a dimer [54]. We previously found that GL analogues SG-1709 and SG-1721 effectively inhibited the phosphorylation of STAT3 in a concentration- and time-dependent manner as compared to GL. SG-1709 and SG-1721 also abrogated JAK1 and JAK2 phosphorylation and inhibited IL-6 or EGF-induced STAT3 activation.

Caspases are a group of protease enzymes that play an essential role in programmed cell death and inflammation [59]. Activation of caspases allows cell death to be controlled while minimizing the effect of cellular components on surrounding tissues [60]. We found that SG-1721 significantly increased cleavage of caspase-3 and PARP than GL. We demonstrated that SG-1721 inhibits the expression of various oncogenic proteins controlled by STAT3 activation. These results correlated with increased apoptosis, as demonstrated upon the drug exposure in TNBC cells. Previous reports have also shown that GL can affect different oncogenic signaling pathways in prostate cancer DU145 cells, thereby resulting in cell cycle arrest [61]. We also found that SG-1721 can induce cell cycle arrest at the $S$ phase in TNBC cells.

Combination therapy can be used to reduce the likelihood of tumors becoming resistant to treatment and has the advantage of affecting multiple targets simultaneously during the treatment process [62]. We demonstrated the anticancer effect of low-dose SG-1721 and radiation combination therapy and identified a novel mechanism mediated by abrogation of STAT3 activation through which the SG-1721 analogue can sensitize TNBC cells to radiation. Finally, a significant anticancer effect of SG-1721 was demonstrated in breast cancer xenograft mouse models. Overall, SG-1721 has been 
conclusively found to significantly inhibit tumor growth without exhibiting any side effects and negatively regulate STAT3 activation in TNBC models.

Author Contributions: H.K. and J.H.L.: Data Curation and formal analysis; H.S.K., T.K., Y.T.H. and Y.G.S.: Design and synthesis of analogues; J.C., Y.G.S., Y.S.K. and K.S.A.: Editing manuscript, K.S.A.: Formal analysis and supervision; Y.S.K. and K.S.A.: Supervision and writing original draft.

Funding: This work was supported by a National Research Foundation of Korea (NRF) grant funded by the Korean government (MSIP) (NRF-2017R1A6A3A11031224 and 2018R1D1A1B07042969).

Conflicts of Interest: The authors declare that they have no competing interests.

\section{References}

1. Hortobagyi, G.N.; de la Garza Salazar, J.; Pritchard, K.; Amadori, D.; Haidinger, R.; Hudis, C.A.; Khaled, H.; Liu, M.C.; Martin, M.; Namer, M.; et al. The global breast cancer burden: Variations in epidemiology and survival. Clin. Breast Cancer 2005, 6, 391-401. [CrossRef] [PubMed]

2. Wang, C.; Kar, S.; Lai, X.; Cai, W.; Arfuso, F.; Sethi, G.; Lobie, P.E.; Goh, B.C.; Lim, L.H.K.; Hartman, M.; et al. Triple negative breast cancer in Asia: An insider's view. Cancer Treat. Rev. 2018, 62, 29-38. [CrossRef] [PubMed]

3. Shanmugam, M.K.; Ahn, K.S.; Hsu, A.; Woo, C.C.; Yuan, Y.; Tan, K.H.B.; Chinnathambi, A.; Alahmadi, T.A.; Alharbi, S.A.; Koh, A.P.F.; et al. Thymoquinone Inhibits Bone Metastasis of Breast Cancer Cells Through Abrogation of the CXCR4 Signaling Axis. Front. Pharmacol. 2018, 9, 1294. [CrossRef] [PubMed]

4. Liu, L.; Ahn, K.S.; Shanmugam, M.K.; Wang, H.; Shen, H.; Arfuso, F.; Chinnathambi, A.; Alharbi, S.A.; Chang, Y.; Sethi, G.; et al. Oleuropein induces apoptosis via abrogating NF-kappaB activation cascade in estrogen receptor-negative breast cancer cells. J. Cell. Biochem. 2018. [CrossRef]

5. Chen, L.; Yuan, Y.; Kar, S.; Kanchi, M.M.; Arora, S.; Kim, J.E.; Koh, P.F.; Yousef, E.; Samy, R.P.; Shanmugam, M.K.; et al. PPARgamma Ligand-induced Annexin A1 Expression Determines Chemotherapy Response via Deubiquitination of Death Domain Kinase RIP in Triple-negative Breast Cancers. Mol. Cancer Ther. 2017, 16, 2528-2542. [CrossRef]

6. Mohan, C.D.; Srinivasa, V.; Rangappa, S.; Mervin, L.; Mohan, S.; Paricharak, S.; Baday, S.; Li, F.; Shanmugam, M.K.; Chinnathambi, A.; et al. Trisubstituted-Imidazoles Induce Apoptosis in Human Breast Cancer Cells by Targeting the Oncogenic PI3K/Akt/mTOR Signaling Pathway. PLoS ONE 2016, 11, e0153155. [CrossRef] [PubMed]

7. Bray, F.; Ferlay, J.; Soerjomataram, I.; Siegel, R.L.; Torre, L.A.; Jemal, A. Global cancer statistics 2018: GLOBOCAN estimates of incidence and mortality worldwide for 36 cancers in 185 countries. CA Cancer J. Clin. 2018, 68, 394-424. [CrossRef]

8. Jia, L.Y.; Shanmugam, M.K.; Sethi, G.; Bishayee, A. Potential role of targeted therapies in the treatment of triple-negative breast cancer. Anti-Cancer Drugs 2016, 27, 147-155. [CrossRef]

9. Bharathkumar, H.; Mohan, C.D.; Ananda, H.; Fuchs, J.E.; Li, F.; Rangappa, S.; Surender, M.; Bulusu, K.C.; Girish, K.S.; Sethi, G.; et al. Microwave-assisted synthesis, characterization and cytotoxic studies of novel estrogen receptor alpha ligands towards human breast cancer cells. Bioorg. Med. Chem. Lett. 2015, 25, 1804-1807. [CrossRef]

10. Shin, E.M.; Hay, H.S.; Lee, M.H.; Goh, J.N.; Tan, T.Z.; Sen, Y.P.; Lim, S.W.; Yousef, E.M.; Ong, H.T.; Thike, A.A.; et al. DEAD-box helicase DP103 defines metastatic potential of human breast cancers. J. Clin. Investig. 2014, 124, 3807-3824. [CrossRef]

11. Woo, C.C.; Hsu, A.; Kumar, A.P.; Sethi, G.; Tan, K.H. Thymoquinone inhibits tumor growth and induces apoptosis in a breast cancer xenograft mouse model: The role of p38 MAPK and ROS. PLoS ONE 2013, 8, e75356. [CrossRef]

12. Manu, K.A.; Shanmugam, M.K.; Rajendran, P.; Li, F.; Ramachandran, L.; Hay, H.S.; Kannaiyan, R.; Swamy, S.N.; Vali, S.; Kapoor, S.; et al. Plumbagin inhibits invasion and migration of breast and gastric cancer cells by downregulating the expression of chemokine receptor CXCR4. Mol. Cancer 2011, 10, 107. [CrossRef]

13. Woo, C.C.; Loo, S.Y.; Gee, V.; Yap, C.W.; Sethi, G.; Kumar, A.P.; Tan, K.H. Anticancer activity of thymoquinone in breast cancer cells: Possible involvement of PPAR-gamma pathway. Biochem. Pharmacol. 2011, 82, 464-475. [CrossRef] [PubMed] 
14. Chua, A.W.; Hay, H.S.; Rajendran, P.; Shanmugam, M.K.; Li, F.; Bist, P.; Koay, E.S.; Lim, L.H.; Kumar, A.P.; Sethi, G. Butein downregulates chemokine receptor CXCR4 expression and function through suppression of NF-kappaB activation in breast and pancreatic tumor cells. Biochem. Pharmacol. 2010, 80, 1553-1562. [CrossRef]

15. Kannaiyan, R.; Manu, K.A.; Chen, L.; Li, F.; Rajendran, P.; Subramaniam, A.; Lam, P.; Kumar, A.P.; Sethi, G. Celastrol inhibits tumor cell proliferation and promotes apoptosis through the activation of c-Jun N-terminal kinase and suppression of PI3 K/Akt signaling pathways. Apoptosis 2011, 16, 1028-1041. [CrossRef]

16. Jemal, A.; Siegel, R.; Xu, J.; Ward, E. Cancer statistics, 2010. CA Cancer J. Clin. 2010, 60, 277-300. [CrossRef] [PubMed]

17. Sorlie, T.; Perou, C.M.; Tibshirani, R.; Aas, T.; Geisler, S.; Johnsen, H.; Hastie, T.; Eisen, M.B.; van de Rijn, M.; Jeffrey, S.S.; et al. Gene expression patterns of breast carcinomas distinguish tumor subclasses with clinical implications. Proc. Natl. Acad. Sci. USA 2001, 98, 10869-10874. [CrossRef]

18. Effi, A.B.; Aman, N.A.; Koui, B.S.; Koffi, K.D.; Traore, Z.C.; Kouyate, M. Breast Cancer Molecular Subtypes Defined by ER/PR and HER2 Status: Association with Clinicopathologic Parameters in Ivorian Patients. APJCP 2016, 17, 1973-1978. [CrossRef]

19. Jin, J.; Zhang, W.; Ji, W.; Yang, F.; Guan, X. Predictive biomarkers for triple negative breast cancer treated with platinum-based chemotherapy. Cancer Biol. Ther. 2017, 18, 369-378. [CrossRef] [PubMed]

20. Hughes, K.; Watson, C.J. The Multifaceted Role of STAT3 in Mammary Gland Involution and Breast Cancer. Int. J. Mol. Sci. 2018, 19, 1695. [CrossRef]

21. Huang, T.T.; Su, J.C.; Liu, C.Y.; Shiau, C.W.; Chen, K.F. Alteration of SHP-1/p-STAT3 Signaling: A Potential Target for Anticancer Therapy. Int. J. Mol. Sci. 2017, 18, 1234. [CrossRef]

22. Laudisi, F.; Cherubini, F.; Monteleone, G.; Stolfi, C. STAT3 Interactors as Potential Therapeutic Targets for Cancer Treatment. Int. J. Mol. Sci. 2018, 19, 1787. [CrossRef]

23. Wu, P.; Wu, D.; Zhao, L.; Huang, L.; Shen, G.; Huang, J.; Chai, Y. Prognostic role of STAT3 in solid tumors: A systematic review and meta-analysis. Oncotarget 2016, 7, 19863-19883. [CrossRef] [PubMed]

24. Banerjee, K.; Resat, H. Constitutive activation of STAT3 in breast cancer cells: A review. Int. J. Cancer 2016, 138, 2570-2578. [CrossRef]

25. Siveen, K.S.; Sikka, S.; Surana, R.; Dai, X.; Zhang, J.; Kumar, A.P.; Tan, B.K.; Sethi, G.; Bishayee, A. Targeting the STAT3 signaling pathway in cancer: Role of synthetic and natural inhibitors. Biochim. Biophys. Acta 2014, 1845, 136-154. [CrossRef] [PubMed]

26. Deng, X.S.; Wang, S.; Deng, A.; Liu, B.; Edgerton, S.M.; Lind, S.E.; Wahdan-Alaswad, R.; Thor, A.D. Metformin targets Stat3 to inhibit cell growth and induce apoptosis in triple-negative breast cancers. Cell Cycle 2012, 11, 367-376. [CrossRef]

27. Lee, M.; Hirpara, J.L.; Eu, J.Q.; Sethi, G.; Wang, L.; Goh, B.C.; Wong, A.L. Targeting STAT3 and oxidative phosphorylation in oncogene-addicted tumors. Redox Biol. 2018. [CrossRef] [PubMed]

28. Arora, L.; Kumar, A.P.; Arfuso, F.; Chng, W.J.; Sethi, G. The Role of Signal Transducer and Activator of Transcription 3 (STAT3) and Its Targeted Inhibition in Hematological Malignancies. Cancers 2018, 10, 327. [CrossRef] [PubMed]

29. Lee, J.H.; Kim, C.; Lee, S.G.; Sethi, G.; Ahn, K.S. Ophiopogonin D, a Steroidal Glycoside Abrogates STAT3 Signaling Cascade and Exhibits Anti-Cancer Activity by Causing GSH/GSSG Imbalance in Lung Carcinoma. Cancers 2018, 10, 427. [CrossRef] [PubMed]

30. Wong, A.L.A.; Hirpara, J.L.; Pervaiz, S.; Eu, J.Q.; Sethi, G.; Goh, B.C. Do STAT3 inhibitors have potential in the future for cancer therapy? Expert Opin. Investig. Drug 2017, 26, 883-887. [CrossRef] [PubMed]

31. Lee, J.H.; Kim, C.; Baek, S.H.; Ko, J.H.; Lee, S.G.; Yang, W.M.; Um, J.Y.; Sethi, G.; Ahn, K.S. Capsazepine inhibits JAK/STAT3 signaling, tumor growth, and cell survival in prostate cancer. Oncotarget 2017, 8, 17700-17711. [CrossRef]

32. Zhang, J.; Ahn, K.S.; Kim, C.; Shanmugam, M.K.; Siveen, K.S.; Arfuso, F.; Samym, R.P.; Deivasigamanim, A.; Lim, L.H.; Wang, L.; et al. Nimbolide-Induced Oxidative Stress Abrogates STAT3 Signaling Cascade and Inhibits Tumor Growth in Transgenic Adenocarcinoma of Mouse Prostate Model. Antioxid. Redox Sign. 2016, 24, 575-589. [CrossRef]

33. Chai, E.Z.; Shanmugam, M.K.; Arfuso, F.; Dharmarajan, A.; Wang, C.; Kumar, A.P.; Samy, R.P.; Lim, L.H.; Wang, L.; Goh, B.C.; et al. Targeting transcription factor STAT3 for cancer prevention and therapy. Pharmacol. Therapeut. 2016, 162, 86-97. [CrossRef] 
34. Subramaniam, A.; Shanmugam, M.K.; Ong, T.H.; Li, F.; Perumal, E.; Chen, L.; Vali, S.; Abbasi, T.; Kapoor, S.; Ahn, K.S.; et al. Emodin inhibits growth and induces apoptosis in an orthotopic hepatocellular carcinoma model by blocking activation of STAT3. Br. J. Pharmacol. 2013, 170, 807-821. [CrossRef]

35. Subramaniam, A.; Shanmugam, M.K.; Perumal, E.; Li, F.; Nachiyappan, A.; Dai, X.; Swamy, S.N.; Ahn, K.S.; Kumar, A.P.; Tan, B.K.; et al. Potential role of signal transducer and activator of transcription (STAT)3 signaling pathway in inflammation, survival, proliferation and invasion of hepatocellular carcinoma. Biochim. Biophys. Acta 2013, 1835, 46-60. [CrossRef] [PubMed]

36. Jung, Y.Y.; Lee, J.H.; Nam, D.; Narula, A.S.; Namjoshi, O.A.; Blough, B.E.; Um, J.Y.; Sethi, G.; Ahn, K.S. Anti-myeloma Effects of Icariin Are Mediated Through the Attenuation of JAK/STAT3-Dependent Signaling Cascade. Front. Pharmacol. 2018, 9, 531. [CrossRef]

37. Kim, C.; Lee, S.G.; Yang, W.M.; Arfuso, F.; Um, J.Y.; Kumar, A.P.; Bian, J.; Sethi, G.; Ahn, K.S. Formononetin-induced oxidative stress abrogates the activation of STAT3/5 signaling axis and suppresses the tumor growth in multiple myeloma preclinical model. Cancer Lett. 2018, 431, 123-141. [CrossRef] [PubMed]

38. Ara, T.; Declerck, Y.A. Interleukin-6 in bone metastasis and cancer progression. Eur. J. Cancer 2010, 46, $1223-1231$. [CrossRef]

39. Yadav, B.S.; Chanana, P.; Jhamb, S. Biomarkers in triple negative breast cancer: A review. J. Clin. Oncol. 2015, 6, 252-263. [CrossRef] [PubMed]

40. Marotta, L.L.; Almendro, V.; Marusyk, A.; Shipitsin, M.; Schemme, J.; Walker, S.R.; Bloushtain-Qimron, N.; Kim, J.J.; Choudhury, S.A.; Maruyama, R.; et al. The JAK2/STAT3 signaling pathway is required for growth of CD44(+)CD24(-) stem cell-like breast cancer cells in human tumors. J. Clin. Investig. 2011, 121, 2723-2735. [CrossRef] [PubMed]

41. Chun, J.; Li, R.J.; Cheng, M.S.; Kim, Y.S. Alantolactone selectively suppresses STAT3 activation and exhibits potent anticancer activity in MDA-MB-231 cells. Cancer Lett. 2015, 357, 393-403. [CrossRef]

42. Weidler, M.; Rether, J.; Anke, T.; Erkel, G. Inhibition of interleukin-6 signaling by galiellalactone. FEBS Lett. 2000, 484, 1-6. [CrossRef]

43. Deorukhkar, A.; Krishnan, S.; Sethi, G.; Aggarwal, B.B. Back to basics: How natural products can provide the basis for new therapeutics. Expert Opin. Investig. Drug 2007, 16, 1753-1773. [CrossRef]

44. Yang, S.F.; Weng, C.J.; Sethi, G.; Hu, D.N. Natural bioactives and phytochemicals serve in cancer treatment and prevention. Evid. Based Complement. Alternat. Med. 2013, 2013, 698190. [CrossRef] [PubMed]

45. Tang, C.H.; Sethi, G.; Kuo, P.L. Novel medicines and strategies in cancer treatment and prevention. BioMed Res. Int. 2014, 2014, 474078. [CrossRef] [PubMed]

46. Hsieh, Y.S.; Yang, S.F.; Sethi, G.; Hu, D.N. Natural bioactives in cancer treatment and prevention. BioMed Res. Int. 2015, 2015, 182835. [CrossRef]

47. Yarla, N.S.; Bishayee, A.; Sethi, G.; Reddanna, P.; Kalle, A.M.; Dhananjaya, B.L.; Dowluru, K.S.; Chintala, R.; Duddukuri, G.R. Targeting arachidonic acid pathway by natural products for cancer prevention and therapy. Semin. Cancer Biol. 2016, 40-41, 48-81. [CrossRef] [PubMed]

48. Don-Doncow, N.; Escobar, Z.; Johansson, M.; Kjellstrom, S.; Garcia, V.; Munoz, E.; Sterner, O.; Bjartell, A.; Hellsten, R. Galiellalactone is a direct inhibitor of the transcription factor STAT3 in prostate cancer cells. J. Biol. Chem. 2014, 289, 15969-15978. [CrossRef]

49. Rudolph, K.; Serwe, A.; Erkel, G. Inhibition of TGF-beta signaling by the fungal lactones (S)-curvularin, dehydrocurvularin, oxacyclododecindione and galiellalactone. Cytokine 2013, 61, 285-296. [CrossRef]

50. Gidlof, R.; Johansson, M.; Sterner, O. Tandem Pd-catalyzed carbonylation and intramolecular vinyl allene Diels-Alder reaction toward galiellalactone analogues. Org. Lett. 2010, 12, 5100-5103. [CrossRef]

51. Kim, H.S.; Kim, T.; Ko, H.; Lee, J.; Kim, Y.S.; Suh, Y.G. Identification of galiellalactone-based novel STAT3-selective inhibitors with cytotoxic activities against triple-negative breast cancer cell lines. Bioorg. Med. Chem. 2017, 25, 5032-5040. [CrossRef]

52. Chun, J.; Tosun, A.; Kim, Y.S. Anti-inflammatory effect of corymbocoumarin from Seseli gummiferum subsp. corymbosum through suppression of NF-kappaB signaling pathway and induction of HO-1 expression in LPS-stimulated RAW 264.7 cells. Int. Immunopharmacol. 2016, 31, 207-215. [CrossRef]

53. Chou, T.C.; Talalay, P. Quantitative analysis of dose-effect relationships: The combined effects of multiple drugs or enzyme inhibitors. Adv. Enzym. Regul. 1984, 22, 27-55. [CrossRef] 
54. Lee, J.H.; Chiang, S.Y.; Nam, D.; Chung, W.S.; Lee, J.; Na, Y.S.; Sethi, G.; Ahn, K.S. Capillarisin inhibits constitutive and inducible STAT3 activation through induction of SHP-1 and SHP-2 tyrosine phosphatases. Cancer Lett. 2014, 345, 140-148. [CrossRef] [PubMed]

55. Levy, D.E.; Inghirami, G. STAT3: A multifaceted oncogene. Proc. Natl. Acad. Sci. USA 2006, 103, 10151-10152. [CrossRef] [PubMed]

56. Sherry, M.M.; Reeves, A.; Wu, J.K.; Cochran, B.H. STAT3 is required for proliferation and maintenance of multipotency in glioblastoma stem cells. Stem Cells 2009, 27, 2383-2392. [CrossRef]

57. Wang, Y.; van Boxel-Dezaire, A.H.; Cheon, H.; Yang, J.; Stark, G.R. STAT3 activation in response to IL-6 is prolonged by the binding of IL-6 receptor to EGF receptor. Proc. Natl. Acad. Sci. USA 2013, 110, 16975-16980. [CrossRef]

58. Paul, M.K.; Mukhopadhyay, A.K. Tyrosine kinase-Role and significance in Cancer. Int. J. Med. Sci. 2004, 1, 101-115. [CrossRef]

59. Perry, D.K.; Smyth, M.J.; Stennicke, H.R.; Salvesen, G.S.; Duriez, P.; Poirier, G.G.; Hannun, Y.A. Zinc is a potent inhibitor of the apoptotic protease, caspase-3. A novel target for zinc in the inhibition of apoptosis. J. Biol. Chem. 1997, 272, 18530-18533. [CrossRef]

60. Parrish, A.B.; Freel, C.D.; Kornbluth, S. Cellular mechanisms controlling caspase activation and function. CSH Perspect. Biol. 2013, 5. [CrossRef]

61. Garcia, V.; Lara-Chica, M.; Cantarero, I.; Sterner, O.; Calzado, M.A.; Munoz, E. Galiellalactone induces cell cycle arrest and apoptosis through the ATM/ATR pathway in prostate cancer cells. Oncotarget 2016, 7, 4490-4506. [CrossRef] [PubMed]

62. Khdair, A.; Chen, D.; Patil, Y.; Ma, L.; Dou, Q.P.; Shekhar, M.P.; Panyam, J. Nanoparticle-mediated combination chemotherapy and photodynamic therapy overcomes tumor drug resistance. J. Control. Release 2010, 141, 137-144. [CrossRef] [PubMed]

Sample Availability: Samples of the compounds are not available from the authors.

(C) 2019 by the authors. Licensee MDPI, Basel, Switzerland. This article is an open access article distributed under the terms and conditions of the Creative Commons Attribution (CC BY) license (http://creativecommons.org/licenses/by/4.0/). 